\title{
Mitogen-activated protein kinase phosphatase-3 (MKP-3) in the surgical wound is necessary for the resolution of postoperative pain in mice
}

This article was published in the following Dove Press journal:

Journal of Pain Research

28 March 2017

Number of times this article has been viewed

\author{
Sladjana Skopelja-Gardner ${ }^{1, *}$ \\ Madhurima Saha ${ }^{1, *}$ \\ Perla Abigail \\ Alvarado-Vazquez ${ }^{2}$ \\ Brenna S Liponis' \\ Elena Martinez' \\ E Alfonso \\ Romero-Sandoval ${ }^{2}$
}

'Department of Anesthesiology, Geisel School of Medicine at Dartmouth, Lebanon, $\mathrm{NH},{ }^{2}$ Department of Pharmaceutical and Administrative Sciences, Presbyterian College School of Pharmacy, Clinton, SC, USA

*These authors contributed equally to this work.
Correspondence: E Alfonso RomeroSandoval

Department of Pharmaceutical and Administrative Sciences, Presbyterian College School of Pharmacy, $307 \mathrm{~N}$.

Broad St, Clinton, SC 29325, USA

Tel +l 8649383833

Fax +l 8649383903

Email asandoval@presby.edu

\begin{abstract}
Mitogen-activated protein kinase (MAPK) phosphatase-3 (MKP-3) and its substrates (extracellular signal-regulated kinase [ERK] and p38) play an important role in pathophysiological mechanisms of acute postoperative and chronic neuropathic pain in the spinal cord. This study aimed to understand the role of MKP-3 and its target MAPKs at the site of surgical incision in nociceptive behavior. Wild-type (WT) and MKP-3 knockout (KO) mice underwent unilateral plantar hind paw incision. Mechanical allodynia was assessed by using von Frey filaments. Peripheral ERK-1/2 and p38 phosphorylation were measured by Western blot. Cell infiltration was determined using hematoxylin and eosin histological staining. Peripheral phosphorylated ERK-1/2 (p-ERK-1/2) inhibition was performed in MKP-3 KO mice. In WT mice, mechanical hypersensitivity was observed on postoperative day $1(0.69 \pm 0.17 \mathrm{~g}$ baseline vs $0.13 \pm 0.08 \mathrm{~g}$ day 1$)$, which resolved normally by postoperative day $12(0.46 \pm 0.08 \mathrm{~g}, \mathrm{~N}=6)$. In MKP-3 KO mice, this hypersensitivity persisted at least 12 days after surgery $(0.19 \pm 0.06 \mathrm{~g} ; \mathrm{N}=6)$. KO mice displayed higher numbers of infiltrating cells $\left(51.4 \pm 6\right.$ cells $\left./ 0.1 \mathrm{~mm}^{2}\right)$ than WT mice $\left(8.7 \pm 1.2\right.$ cells $\left./ 0.1 \mathrm{~mm}^{2}\right)$ on postoperative day 1 (vs $5-6$ cells $/ 0.1 \mathrm{~mm}^{2}$ at baseline) that returned to baseline 12 days after surgery (10-12 cells/ $0.1 \mathrm{~mm}^{2}$ ). In WT mice, peripheral p-p38 and p-ERK-1/2 expression increased (5- and 3-fold, respectively) on postoperative days 1 and 5, and returned to basal levels 7-12 days after surgery ( $\mathrm{N}=3$ per group). Peripheral p-p38 levels in MKP-3 KO mice followed a similar expression pattern as WT mice. Peripheral p-ERK-1/2 levels in MKP-3 KO mice remained elevated 12 days after surgery (2.5-fold, $\mathrm{N}=3$ per group). Administration of PD98059 (MEK inhibitor, $\mathrm{N}=8$, vehicle $\mathrm{N}=9$ ) reduced $\mathrm{p}-\mathrm{ERK}-1 / 2$ expression in the incised tissue and blocked hypersensitivity in MKP-3 KO mice $(\mathrm{N}=6)$. The findings of this study suggest that MKP-3 is pivotal for normal resolution of acute postoperative allodynia, through the regulation of peripheral p-ERK-1/2.
\end{abstract}

Keywords: DUSP-6, p38, ERK

\section{Introduction}

Although effective treatment can resolve acute postsurgical pain in many patients, $30 \%-50 \%$ of individuals who undergo common invasive surgeries suffer persistent postoperative pain. ${ }^{1}$ The nociceptive processes under these conditions are not fully understood, and our knowledge on the triggers of persistent postsurgical pain is limited. Identifying these factors (ie, targets) will aid the development of more adequate non-invasive therapies. ${ }^{2}$

Studies of pain axes in the spinal cord have shown that mitogen-activated protein kinases (MAPKs) contribute to the initiation and maintenance of post-surgical pain. 
Inactivation of phosphorylated MAPK p38 (p-p38) successfully reduced pain-related behaviors in rodents, following plantar incision surgery ${ }^{3,4}$ and treated postoperative pain in humans. ${ }^{5}$ The role of MAPKs is also instrumental in chronic pain conditions. Phosphorylation of p38 or extracellular signal-regulated kinase (ERK)-1/2, another MAPK, is necessary for the development and perpetuation of mechanical hypersensitivity, as observed in models of chronic neuropathic $^{6}$ and acute-to-chronic postoperative pain. ${ }^{3,7}$ Although p38 inhibition has demonstrated some efficacy in neuropathic pain in humans, ${ }^{9,10}$ its role in this condition remains unclear. ${ }^{11}$

Even though the pathophysiological events in the spinal cord are important for the maintenance of postoperative pain, ${ }^{3,7,8}$ cellular and molecular processes at the site of the surgical incision are also relevant. Studies on injured tissue in rodents, following paw incision, have linked the up-regulation of neurotrophin and cytokine genes with postoperative pain. ${ }^{12}$ Molecular mediators such as nerve growth factor, ${ }^{13}$ lactic acid, ${ }^{14}$ prostaglandins, ${ }^{15}$ and complement factor, ${ }^{16}$ in the incised tissue are also related to postoperative pain. Moreover, inhibition of p-p38 and phosphorylated ERK$1 / 2$ (p-ERK-1/2), in the mouse peripheral tissue, reduced nociceptive behaviors in a model of phorbol 12-myristate 13-acetate-induced inflammatory pain. ${ }^{17}$ Increased levels of a downstream MAPK signaling product, interleukin 6, at the incision site, are associated with postoperative pain in humans ${ }^{18}$ and with the development of hyperalgesia in murine pain models. ${ }^{19-21}$ This study proposes that a dysregulation of the peripheral mechanisms that promote MAPK deactivation underlies the transition from acute to persistent postoperative pain.

MAPK phosphatases (MKP) are the major negative regulators of MAPKs. Specifically, MKP-3 preferentially dephosphorylates p-ERK-1/2, whereas MKP-1 favors p-p38 dephosphorylation. ${ }^{22,23}$ However, their substrate preference varies between cell types, anatomical locations, or pathological conditions. ${ }^{7,24}$ It has been demonstrated that MKPs can act as potential analgesic targets..$^{25,26}$ This study showed that impaired MKP-3 signaling in the spinal cord leads to persistent mechanical hypersensitivity, through dysregulation of p-p38 and p-ERK-1/2. ${ }^{7}$ Moreover, it demonstrated that spinal induction of MKP-1 prevents neuropathic mechanical hypersensitivity in rats. ${ }^{27}$ In addition to their role in the central nervous system, MKP-1 and MKP-3 mediate peripheral inflammatory processes. $^{28,29}$ However, the contribution of MKPs at the peripheral sites in pain conditions has not been investigated.

This study aimed to understand the role of MKPs and their target MAPKs at the site of the surgical incision in nociceptive behavior in mice. Based on previous studies, ${ }^{7,26}$ it is hypothesized that the dysregulation of peripheral MKP-3 will result in persistent ERK-1/2 and p38 phosphorylation and persistent mechanical allodynia, in the mouse paw incision model.

\section{Materials and methods}

\section{Animals}

Wild-type (WT, B6129SF2/J) and MKP-3 knockout (KO, B6129X1-Dusp6 TM1Jmol/J) male and female (50-60 days old) mice were used (The Jackson Laboratory, Bar Harbor, ME, USA). MKP-3 KO mice were reported to be deficient in MKP-3 protein in the spleen, heart, lungs, and thymus tissues. ${ }^{30}$ It was shown that MKP-3 KO mice lack MKP-3 protein in the spinal cord. ${ }^{7}$ The authors confirmed that these mice also lack MKP-3 in the paw tissue (Figure 1). The animals were housed individually and maintained in light-dark cycles for $12 \mathrm{~h}$ with ad libitum access to food and water. All animal procedures were done in accordance with the Guidelines for Animal Experimentation of the International Association for the Study of Pain and were approved by the Institutional Animal Care and Use Committee at Dartmouth College. All the animals ( $\mathrm{KO}$ or WT) used for behavioral experiments or that received treatment or that were used for tissue collection and molecular assessments were distributed using a randomized allocation to different groups. The method used was simple, choosing random numbers that coincide with the identification number of the animals from a list including all the animals of the given groups.

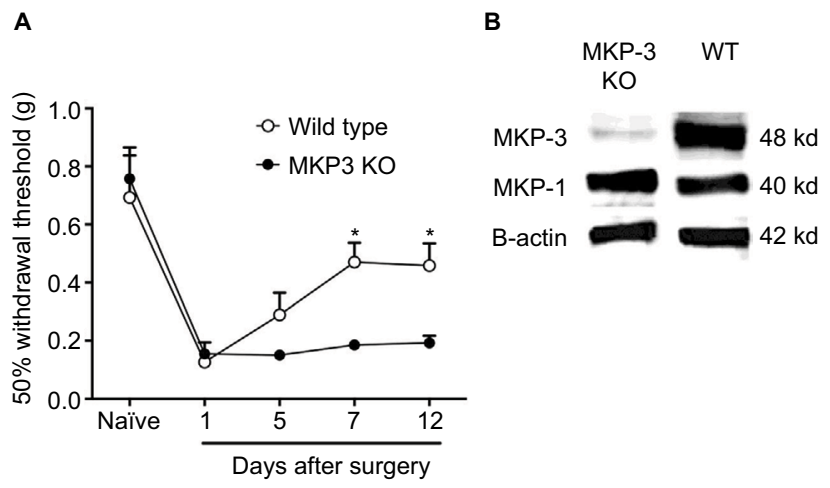

Figure I 50\% paw withdrawal threshold (A) in naïve mice and mice that underwent paw incision surgery, measured on days $1,5,7$, and 12 in wild-type (WT, open circles [0] $n=6$ ) and in MKP-3 KO mice (closed circles [•] $n=6$ ). Data were analyzed using two-way ANOVA + Bonferroni post hoc analysis. $* P<0.05$, WT vs MKP-3 KO. Representative Western blot images (B) of hind paw plantar tissue MKP-3 and MKPI ( $\beta$-actin was used as the loading control) in WT and MKP-3 KO naive mice. Data are expressed as mean \pm SEM.

Abbreviations: WT, wild type; KO, knockout; ANOVA, analysis of variance; MKP-3, Mitogen-activated protein kinase phosphatase-3; SEM, standard error of mean. 


\section{Surgery: paw incision model of postoperative pain}

All the animals were anesthetized with isoflurane (4\% induction, $2 \%$ maintenance) in oxygen, delivered through a nose cone. Plantar aspect of the left hind paw was sterilized with providone-iodine before and after surgeries. Approximately $0.5 \mathrm{~cm}$ longitudinal incision was made through skin and fascia of the plantar aspect of the left hind paw from the heel to the base of the toes, using a No. 10 scalpel blade and sterile technique. The plantaris muscle and ligaments were elevated and stretched for 6-8 s (they were not incised). The wound was cleaned and apposed with inverted 6.0 silk sutures (Ethicon Inc., Somerville, NJ, USA). The animals were allowed to recover from the surgery for $1 \mathrm{~h}$ and maintained with ad libitum access to food and water. ${ }^{31,32}$

\section{Behavioral testing}

Mechanical allodynia, measured as 50\% withdrawal threshold, was assessed in the ipsilateral paw to surgery using von Frey filaments (0.07-, 0.17-, 0.40-, 0.60-, 1.04-, 1.37-, and 2.0-g bending force) (Stoeling, Wood Dale, IL, USA) and calculated using an up-down statistical method., ${ }^{3,33}$ In both WT and KO animal groups, baseline behavior was tested in naïve mice (no surgery), as well as 1, 5, 7, and 12 days after surgery ( $n=6$ per group; 6 males in each group). The animals were followed up until postoperative day 12 since it had been previously shown that paw incision-induced inflammation, measured as paw circumference, returns to baseline levels on day 12 after surgery. ${ }^{7}$ An additional group of MKP-3 KO mice $(n=3)$ was used to assess the role of MAPKs; $50 \%$ withdrawal threshold was measured before the surgery, 12 days after surgery, and $0.5,1$, and $2 \mathrm{~h}$ after intraplantar injection of an MEK inhibitor (PD98059) that blocks phosphorylation of ERK-1/2, administered on day 12 . Two experimenters (blind to treatment conditions) performed two sets of experiments; the data were combined for analysis.

\section{Cellular infiltration}

Animals were anesthetized and perfused transcardially using $0.1 \mathrm{M}$ phosphate-buffered saline, followed by $4 \%$ formaldehyde. Tissue from the operated paw was removed in a consistent manner: the center of the incision wound was localized, and a No. 10 blade was used to cut the soft tissue $1.5 \mathrm{~mm}$ apart from the center, rostrally, and caudally. The plantar bones determined the depth of the collected tissue; the tissue was removed from the paw by an additional longitudinal cut above the bone of the paw. A $3 \mathrm{~mm}$ piece of plantar paw tissue was placed in $30 \%$ sucrose solution for $48-72 \mathrm{~h}$, at $4^{\circ} \mathrm{C}$. The tissue was mounted and frozen in optimal cutting temperature compound (Sakura Finetek, Torrence, CA, USA) at $-80^{\circ} \mathrm{C}$. Hematoxylin and Eosin (H\&E) staining was performed on $20 \mu \mathrm{m}$ paw tissue sections, cut using Leica CM1859 Cryostat, according to standard protocols. The stained tissues were dehydrated and sealed with Vectashield mounting medium (Vector Labs, Burlingame, CA, USA). Images were obtained using Q-fired cooled CCD camera attached to an Olympus microscope BX60. H\&E staining was performed on two groups of animals ( $n=3$ male animals per group, WT and MKP-3 KO), prior to (naïve) and on different days after surgery (days $1,5,7$, and 12). Three tissue sections per animal per time point were evaluated for the number of infiltrating cells. Three areas of $0.1 \mathrm{~mm}^{2}$ per tissue section were scored and averaged. The percentage of polymorphonuclear (PMN) and mononuclear cells was assessed morphologically. One experimenter (blind to genotype conditions) performed this analysis.

\section{Drugs and intraplantar injections}

PD98059, an MEK inhibitor used to block p-ERK-1/2 expression (Sigma-Aldrich Co., St. Louis, MO), was diluted in dimethylsulfoxide and saline (1:1, vehicle). PD98059 $(25 \mathrm{nmol}, 8 \mu \mathrm{L})$, or vehicle, was injected intraplantary to the left hind paw of MKP-3 KO mice (ipsilateral to surgery), on postoperative day 12 . The concentration of the drug was chosen based on a previous study ${ }^{7}$ and other pain mouse models. ${ }^{34}$ Mechanical allodynia was assessed before surgery and on postoperative day 12, prior to PD98059 injection, as well as $0.5,1$, and $2 \mathrm{~h}$ following drug administration. The experimenter performed these studies blind to treatment conditions ( $\mathrm{n}=9$, six males and three females in the vehicle treated group; $n=8$, five males and three females in the MEK inhibitor-treated group). Western blot was performed on six paw tissue samples, five male mice and one female mouse, from each treatment group.

\section{Western blot}

The animals were euthanized using decapitation under isoflurane anesthesia ( $4 \%$ in oxygen). The plantar tissues of the left hind paw (ipsilateral) were removed using the method described in the "Cellular Infiltration" section. A $3 \mathrm{~mm}$ piece of tissue was obtained and processed; tissue was sonicated in PBS, with 1:1000 protease inhibitors (Sigma-Aldrich Co.). Protein concentrations were determined by DC assay (BioRad Laboratories Inc., Hercules, CA, USA). A $50 \mu \mathrm{g}$ protein 
sample was loaded onto sodium dodecyl sulfate (SDS) polyacrylamide electrophoresis gel (10\%; Bio-Rad Laboratories Inc.). The protein was transferred to a $0.2 \mu \mathrm{m}$ nitrocellulose membrane (Bio-Rad Laboratories Inc.) and processed as previously described. ${ }^{7}$ Primary antibodies used were as follows: mouse anti-p-ERK-1/2 (1:1000), rabbit anti-p-p38 (1:1000), mouse anti-ERK-1/2 (1:1000), rabbit anti-p38 (1:500) (Cell Signaling Technology, Inc., Danvers, MA, USA), rabbit anti-MKP-1 (1:500; Santa Cruz Biotechnology Inc., Dallas, TX, USA), and rabbit anti-MKP-3 (1:3000; Cell Signaling Technology). $\beta$-Actin was used as loading control and stained using mouse anti- $\beta$-actin primary antibody (1:3000; Abcam, Cambridge, MA, USA). Following primary antibody incubation $\left(16-20 \mathrm{~h}\right.$ at $\left.4^{\circ} \mathrm{C}\right)$, the blots were washed in tris-buffered saline (TBS) $+0.05 \%$ Tween 20 and incubated for $1 \mathrm{~h}$ at room temperature in one of the following secondary antibodies: goat anti-mouse HRP-conjugated antibody (1:3000; Bethyl Laboratories Inc., TX, USA) or goat anti-rabbit horseradish peroxidase (HRP)-conjugated antibody (1:3000; Pierce, Rockford, IL, USA). Subsequently, the blots were washed and visualized using Super Signal West Femto Maximum Sensitivity Substrate (Pierce). Imaging was conducted using Syngene G-Box system and software (Synoptics, Frederick, MD, USA). Following visualization, the membranes were incubated for $25 \mathrm{~min}$ at $37^{\circ} \mathrm{C}$ in $5 \%$ sodium azide TBS buffer and re-probed with another primary and secondary antibody as described previously. ${ }^{7}$ In some cases (MKP-3 and MKP-1 Western blots), $\beta$-actin blots were run simultaneously on a different membrane using the same samples and same protein amounts in both membranes.

Signal intensity of the protein of interest was assessed using Image J software, and the data were quantified by dividing the intensity of the signal of the protein of interest with the corresponding signal intensity of $\beta$-actin. Quantifications of p-ERK-1/2 and p-p38 levels were done by normalizing p-ERK-1/2, ERK-1/2, p-p38, and p38 against $\beta$-actin, after which p-ERK-1/2 and p-p38 data were quantified as relative intensity of the particular signal, divided by the previously normalized value of total ERK-1/2 and total p38, respectively. For the MKP-1, MKP-3, p-ERK, and p-p38 time course quantification studies in WT and MKP-3 KO groups, two independent experimenters (blind to genotype conditions) performed these analyses, and both the sets of data were combined ( $\mathrm{n}=3$ male mice per group).

\section{Statistical analysis}

Statistical analyses were performed using GraphPad Prism 5 (GraphPad Software Inc., La Jolla, CA, USA), and all data are expressed as mean \pm standard error of mean (SEM). The size of the samples was decided based on previous publications by these authors, and therefore, no a priori statistical power calculation was conducted. ${ }^{7,24}$ Normality was tested using Shapiro-Wilk test. Two data sets from the MEK inhibitor (PD98059) group and one data set from the vehicle group were identified as outliers (2-fold standard deviation) and therefore excluded from the analysis in the behavioral experiments.

Behavior data were analyzed using a repetitive measurement two-way analysis of variance (ANOVA) followed by Bonferroni post hoc test; $\mathrm{N}=6$. The values for cellular infiltration measurements after the paw incision did not follow a normal distribution and were analyzed as nonparametrical data. Kruskal-Wallis followed by a Dunn test was performed to determine the changes in cellular infiltration against the baseline values (naïve mice) in each group. Mann-Whitney $U$ test was performed to compare the effects on cellular infiltration between the $\mathrm{KO}$ and WT groups at each time point; $\mathrm{N}=6$. The Western blot analyses between groups were determined by one-way ANOVA followed by Dunnett's post-test using the naïve group as the control, or two-way ANOVA followed by Bonferroni's post-test, as appropriate, $\mathrm{N}=3$. Comparison of the relative expression of p-ERK-1/2 in PD98059-treated versus vehicle-treated group in $\mathrm{KO}$ mice was performed using Mann-Whitney $U$ test for non-parametrical data $(\mathrm{N}=6)$. A $P$-value $<0.05$ was considered statistically significant.

\section{Results}

\section{Relationship between MKP-3 and pain- related behaviors in the mouse model of acute postoperative pain}

In both WT and MKP-3 KO mice, paw incision surgery led to an equal level of mechanical hypersensitivity on postoperative day 1 (Figure 1A). Although the 50\% withdrawal threshold in WT mice returned to baseline levels on postoperative day 12, mechanical allodynia in MKP-3 KO mice continued until 7-12 days after surgery (Figure 1A).

As previously demonstrated, MKP-3 KO mice did not exhibit overtly altered behavior when compared with WT mice. ${ }^{7}$ Development and grooming were comparable between the two murine strains. It was confirmed that MKP-3 KO mice did not express MKP-3 protein in the paw tissue and that MKP-1 protein expression was consistent between WT and MKP-3 KO mice (Figure 1B), thus excluding potential compensatory mechanisms. 


\section{Role of peripheral inflammation in mechanical allodynia in WT and MKP-3 KO mice}

This study evaluated the cellular infiltration levels in the paw tissue 1-7 days after surgery and compared it with cellularity at baseline and at day 12 after surgery. H\&E histological staining in WT mice showed a significant increase in the number of infiltrating cells on postoperative day 5 after surgery, which remained elevated at day 7 after surgery (Figure 2A and B). In contrast, in MKP-3 KO mice, the number of infiltrating cells significantly increased on postoperative day 1 , in which a peak effect and a significant difference compared to the WT group was observed. The number of cells in the KO mice declined initially but remained significantly elevated on postoperative days 5 and 7 (Figure 2A and B). However, these values were not different from the WT group values (Figure 2A and B). The degree of cellular infiltration returned to basal levels 12 days after surgery in both the groups.

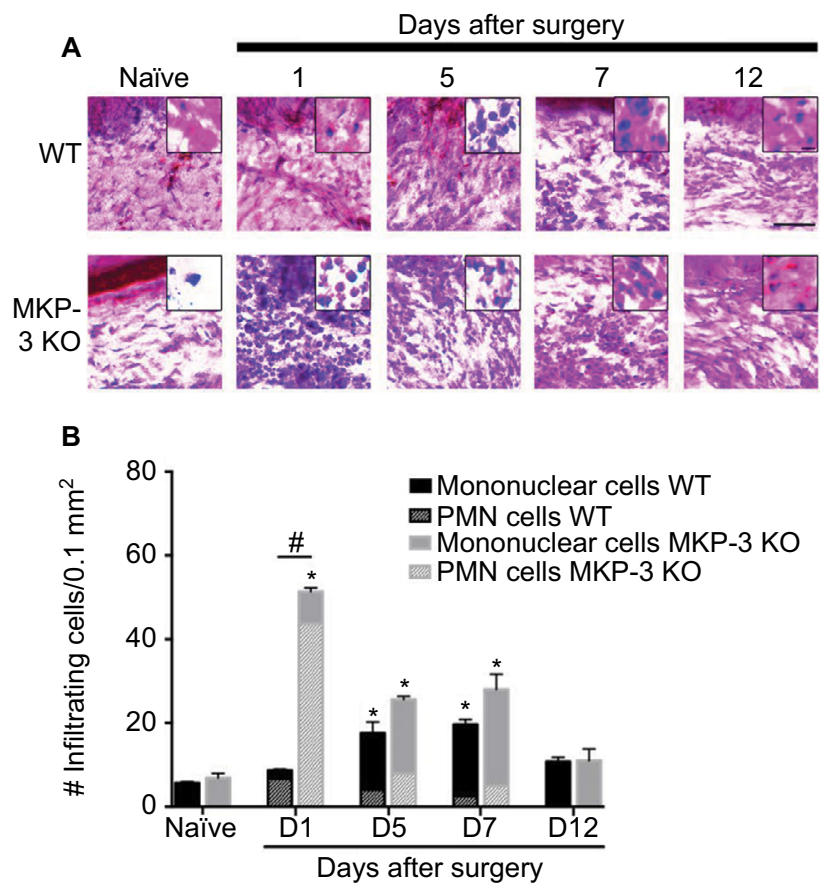

Figure 2 Representative images of H\&E staining (A) (scale bar $=100 \mu \mathrm{m}$ ) of paw tissue ipsilateral to incision of wild-type (WT) and MKP-3 KO mice, in naïve mice (left hind paw plantar tissue) and on days I, 5, 7, and 12 after paw incision surgery. Cellular and nuclear morphology of some cells are shown in magnified inserted images (scale bar $=20 \mu \mathrm{m}$ ). Quantification of the number of infiltrated cells $(\mathbf{B})$ in paw tissue ipsilateral to incision of wild-type (WT) and MKP-3 KO mice, in naïve mice (left hind paw plantar tissue) and on days I, 5, 7, and 12 after paw incision surgery ( $n=3 /$ group). The number of polymorphonuclear cells (line pattern) and mononuclear cells (solid color) is represented within each bar. $* P<0.05$ vs naïve, Kruskal-Wallis tests followed by a Dunn test; ${ }^{*} P<0.05$ WT vs MKP-3 KO, MannWhitney $U$ test. Data are expressed as mean \pm SEM.

Abbreviations: H\&E, hematoxylin and eosin; WT, wild type; KO, knockout; MKP3, Mitogen-activated protein kinase phosphatase-3; PMN, polymorphonuclear cells; SEM, standard error of mean.
When different populations of PMN and mononuclear cells were evaluated, it was found that the percentage of PMN cells in the MKP-3 KO mice $(86.9 \pm 2.8 \%)$ was significantly higher than that in WT mice $(77.1 \pm 5.1 \%) 1$ day after surgery $(P=0.02)$. A decrease in the percentage of PMN cells was found at the later points after paw incision in both MKP-3 KO and WT mice, but no changes in the percent of PMN cells were found between the groups on postoperative days 5 (MKP-3 KO $=31.4 \pm 5.7 \%$, WT $=22.9 \pm 3.3 \%$ PMN cells, $P=0.20$ ) and 7 (MKP-3 KO $=18.5 \pm 5.2 \%$, WT $=13.1 \pm 2.3 \%$ PMN cells, $P=0.10$ ). The proportion of mononuclear cells was smaller than the proportion of PMN cells on postoperative day 1 in both MKP-3 KO and WT mice, as expected. However, a lower percentage of mononuclear cells was obseverd on postoperative day 1 in the MKP-3 KO group (13.1 $\pm 4.0 \%)$ compared to the WT group $(22.9 \pm 5.1 \%$, $P=0.02)$. Conversely, a predominant proportion of mononuclear cells was observed on postoperative days 5 (MKP-3 $\mathrm{KO}=68.6 \pm 5.73 \%$, WT $=77.1 \pm 4.6 \%$ PMN cells, $P=0.14$ ) and 7 (MKP-3 KO $=81.5 \pm 5.2 \%$, WT $=86.9 \pm 2.28 \%$ PMN cells, $P=0.10$ ) in both MKP-3 KO and WT groups, but no significant differences were found between these groups.

\section{p-ERK-I/2 and p-p38 expression levels in the paw tissue of WT and MKP-3 $\mathrm{KO}$ mice}

Since MAPKs are the natural substrates of MKP-3, and MAPKs determine at least in part the pro-inflammatory phenotype of immune cells, the phosphorylation level of p-ERK and p-p38 was studied in the paw soft tissue (surgical area). In WT mice, levels of $\mathrm{p}$-ERK-1/2 protein were significantly elevated on postoperative days 1 and 5, and no significant differences were observed between day 7 or 12 after surgery and the naïve animals (Figure 3A and B). Similarly, expression levels of $\mathrm{p}-\mathrm{p} 38$ protein significantly increased on postoperative days $1-5$, and no statistical differences were observed between day 7 or 12 after surgery and the naïve groups (Figure 3A and C). These observations are consistent with the decrease in paw edema ${ }^{7}$ and cellular infiltrates, as well as with the resolution of mechanical allodynia in WT mice, on days $7-12$ following surgery.

In MKP-3 KO mice, $\mathrm{p}-\mathrm{ERK}-1 / 2$ protein levels were significantly elevated on postoperative day 1 and remained consistently elevated all through postoperative day 12 , relative to the protein levels in naïve MKP-3 KO mice (Figure 3A and D). On the contrary, $\mathrm{p}-\mathrm{p} 38$ expression patterns in MKP-3 KO mice after paw incision surgery resembled those of WT mice, whereas p-p38 levels increased on postoperative days 
A

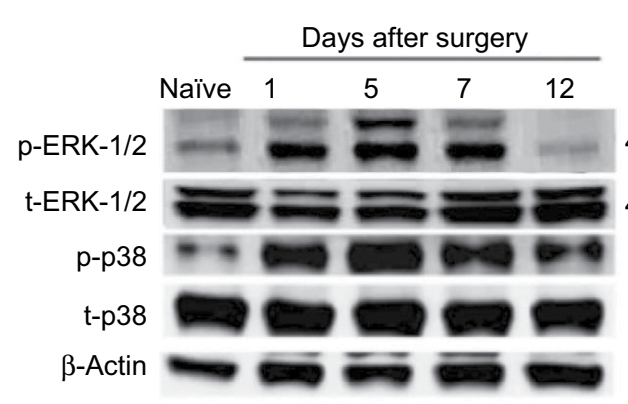

B

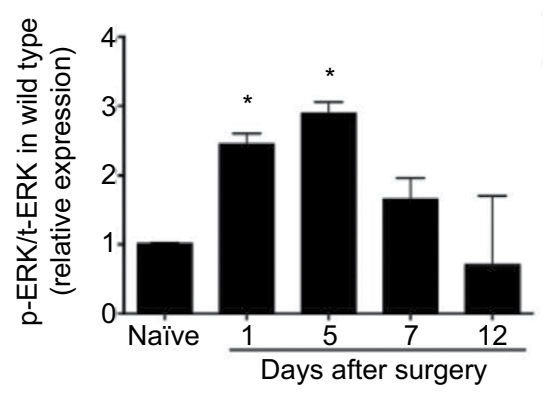

C

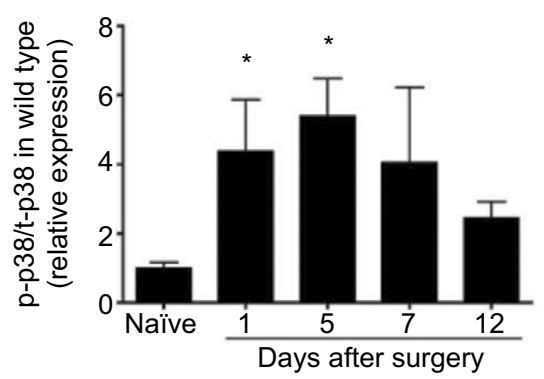

MKP3-KO

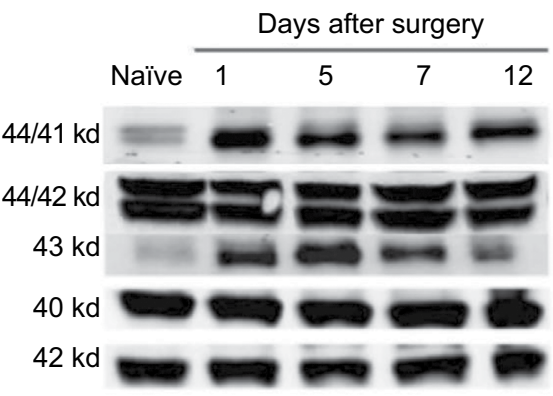

D

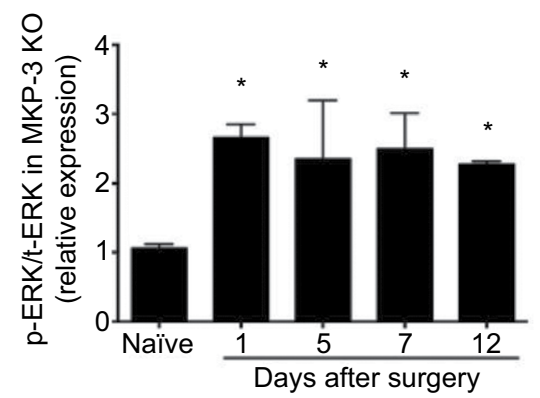

E

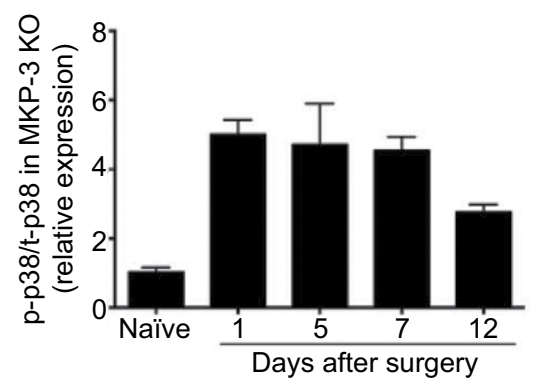

Figure 3 Representative Western blot images of p-ERK-I/2, total-ERK-I/2, p-p38, total-p38 and $\beta$-actin (loading control) from paw tissue of wild-type and MKP-3 KO mice, in naive condition and on days I, 5, 7, and 12 after paw incision surgery (A). Quantification of p-ERK-1/2 (B) and p-p38 (C) expression in the paw tissue of wild-type mice, and p-ERK-I/2 (D) and p-p38 (E) expression in the paw tissue of MKP-3 KO mice, in naïve condition and on days I, 5, 7, and I2 after paw incision surgery ( $\mathrm{n}=3 /$ group). Data were normalized to naïve condition and analyzed using one-way ANOVA + Dunnett's post-test, $* P<0.05$ vs naïve. Data are expressed as mean \pm SEM.

Abbreviations: ERK, extracellular signal-regulated kinase; KO, knockout; MKP-3, Mitogen-activated protein kinase phosphatase-3; ANOVA, analysis of variance; SEM, standard error of mean.

$1-7$; they subsided by day 12 after surgery, relative to naïve mice (Figure $3 \mathrm{~A}$ and $\mathrm{E}$ ).

\section{MKP-3 and MKP-I expression levels in the paw tissue of WT and MKP-3 KO mice}

In order to understand the temporal MAPK dynamics observed in the soft tissue of the operated paw, temporal expression of MKP-3 in WT mice and the expression of MKP-1, the other major regulator of MAPKs, were studied. In WT mice, MKP-3 expression levels in the paw tissue increased on postoperative days 5-7 (Figure 4A and C), in accordance with the declining expression levels of p-ERK-1/2 and p-p38 at these time intervals (Figure 3). Although MKP-1 expression in the WT paw tissue initially declined on postoperative day 1, MKP-1 protein levels increased 7 days after surgery (Figure 4A and D), potentially contributing to the return of p-p38 and p-ERK1/2 expression to basal levels on postoperative day 12 .

In MKP-3 KO mice, MKP-1 expression levels in the paw tissue increased on postoperative day 1 , but returned to baseline levels on days 5-12 after surgery, relative to naïve mice (Figure 4B and D). The difference in MKP-1 expression levels between WT and MKP-3 KO mice, on postoperative day 1 , could be due to a compensatory mechanism in MKP-3 KO mice following paw incision. Interestingly, the high expression of p-p38 on postoperative day 1 is not in 
A

Wild type

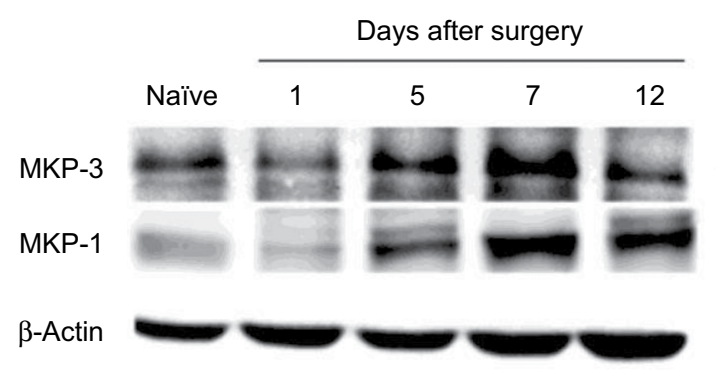

B

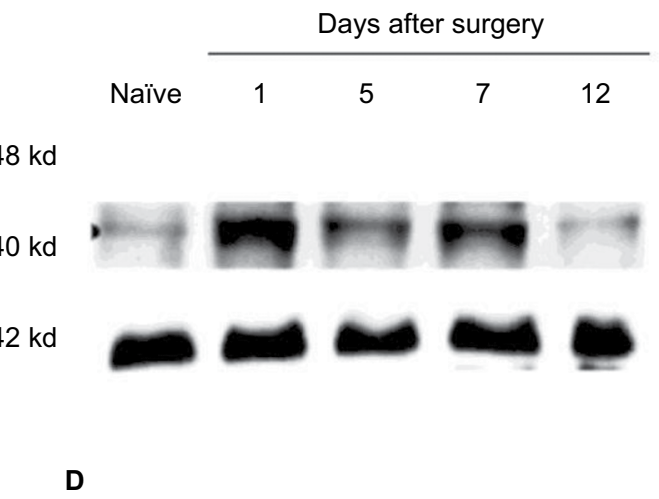

C

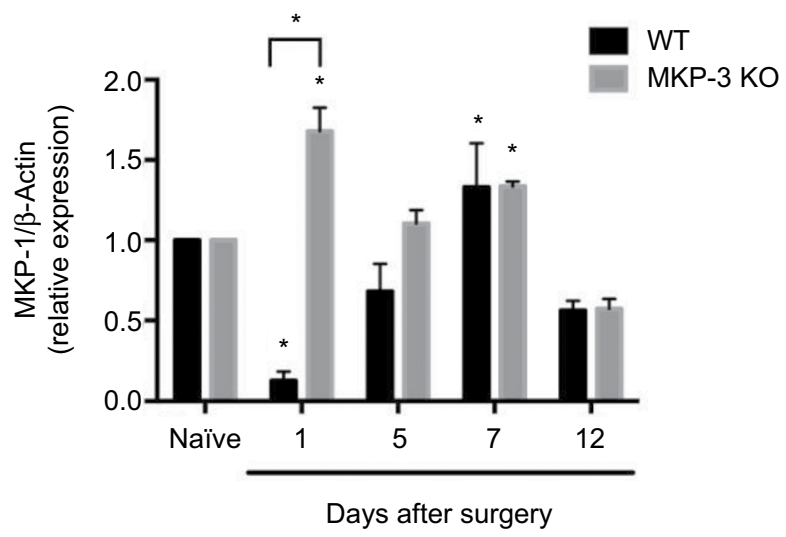

Figure 4 Representative Western blot images of MKP-I, MKP-3, and $\beta$-actin (loading control) from paw tissue of WT mice (A), in naïve condition and on days I, 5, 7, and 12 after paw incision surgery, and representative Western blot images of MKP-I and $\beta$-actin (loading control) from paw tissue of MKP-3 KO mice (B), in naïve condition and on days I, 5, 7, and I 2 after paw incision surgery. Quantification of MKP-3 expression in the paw tissue of WT (C) and of MKP-I expression in the paw tissue of MKP-3 KO mice (D), in naïve condition and on days I, 5, 7, and I 2 after paw incision surgery ( $n=3 /$ group). Data were analyzed using one-way ANOVA + Dunnett's post-test (C) or two-way ANOVA + Bonferroni post hoc (D); $* P<0.05$ vs naïve. Data are expressed as mean \pm SEM.

Abbreviations: WT, wild type; KO, knockout; MKP-3, mitogen-activated protein kinase phosphatase-3; ANOVA, analysis of variance; SEM, standard error of mean.

accordance with the elevation of MKP-1 protein levels in MKP-3 KO mice, despite p-p38 being the primary MKP-1 substrate. This suggests that other factors might contribute to the regulation of p-p38 at the early stages after surgery, for example, MKP-3.

\section{Effects of inhibiting ERK-I/2 phosphorylation in the paw tissue of MKP-3 KO mice}

This study observed that p-ERK-1/2 expression levels remained elevated in the paw tissue of MKP-3 KO mice on postoperative day 12 , contrary to the unchanged p-p38 levels, relative to naïve mice (Figure $3 \mathrm{D}$ and $\mathrm{E}$ ). To test a potential causative association between persistent p-ERK and persistent allodynia, the effect of inhibiting MEK was studied, which subsequently prevents ERK phosphorylation, in the paw tissue, on mechanical allodynia in MKP-3 KO mice, 12 days after surgery.
It was also observed that local intradermal administration of a small molecule MEK inhibitor, PD98059 (25 nmol), partially, but significantly, reversed incision-induced persistent mechanical allodynia $1-2 \mathrm{~h}$ after injection, compared to the vehicle-treated group (Figure 5A). To confirm the inhibitory effects of PD98059 over p-ERK-1/2, its expression levels after treatment were measured. A partial but significant reduction in $\mathrm{p}-\mathrm{ERK}-1 / 2$ expression was observed in the paw tissue $2 \mathrm{~h}$ after treatment, compared to the vehicle (Figure 5B and $\mathrm{C}$ ). These data strongly suggest a causative relationship between peripheral p-ERK expression and persistent postoperative hypersensitivity observed in MKP-3 KO mice.

\section{Discussion}

This study demonstrates that lack of MKP-3 protein in mice leads to a long-lasting phosphorylation of MAPK ERK-1/2 in the peripheral tissue following a surgical intervention (paw incision), which results in persistent postoperative 


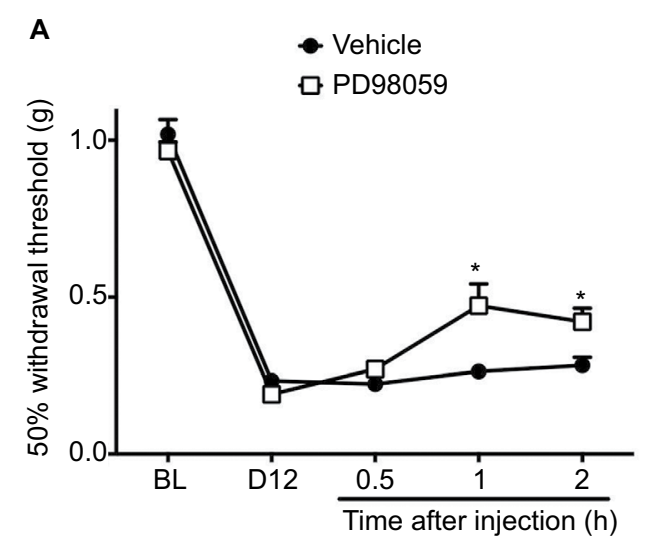

C

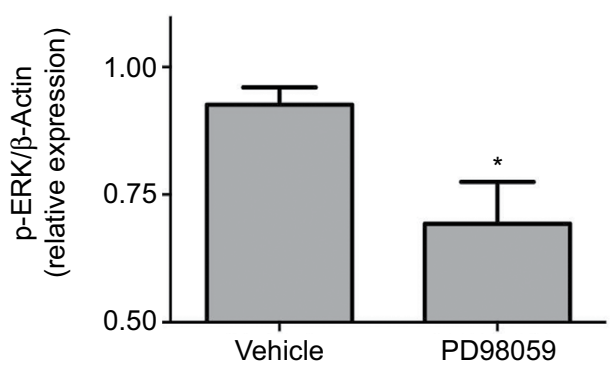

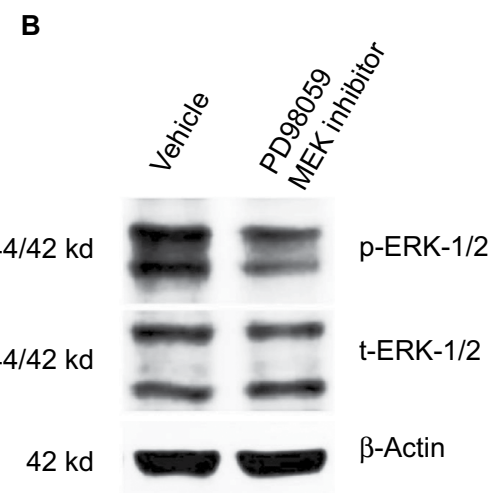

Figure 5 50\% paw withdrawal threshold (A) in MKP-3 KO mice before (baseline, BL) and 12 days following paw incision (DI2), and 0.5, I, and $2 \mathrm{~h}$ following intraplantar administration of $25 \mathrm{nmol}$ PD98059 (MEK-I/2 inhibitor, $n=8$ ) or vehicle $(n=9)$ on postoperative day I2. Data were analyzed using two-way ANOVA + Bonferroni's post hoc analysis. $* P<0.05$ vs vehicle. Representative Western blot images of $\mathrm{p}$-ERK-I/2, total-ERK-I/2, and $\beta$-actin (loading control) (B) from paw tissue of MKP-3 KO mice (postoperative day 12) $2 \mathrm{~h}$ following intraplantar administration of PD98059 or vehicle. Quantification of p-ERK-I/2 expression (C) in paw tissue of MKP-3 KO mice (postoperative day 12) $2 \mathrm{~h}$ following intraplantar administration of PD98059 or vehicle ( $\mathrm{n}=6$ per group, five male mice and one female mouse, from each treatment group). Data were analyzed using one-way ANOVA + Bonferroni's post hoc analysis. $* P<0.05$ versus vehicle. Data are expressed as mean $\pm S E M$.

Abbreviations: WT, wild type; KO, knockout; ERK, extracellular signal-regulated kinase; MKP-3, mitogen-activated protein kinase phosphatase-3; ANOVA, analysis of variance; SEM, standard error of mean.

mechanical allodynia. The major novelty of this study is therefore the discovery of the important role that MKP-3 plays in the periphery in the transition from acute to chronic postoperative mechanical hypersensitivity.

It has been previously shown that dysregulation of MKPs in the spinal cord is associated with chronic neuropathic pain, such that MKP-1 and MKP-3 induction in the spinal cord reduced mechanical allodynia in the rat model of neuropathic pain. ${ }^{26,27}$ In a more recent study, a mouse model of acute postoperative pain was used, that is, paw incision surgery, to demonstrate that MKP-3 also plays a role in the transition from acute to chronic postoperative mechanical allodynia through mechanisms in the spinal cord. ${ }^{7}$ Using the same model, this study aimed to understand the role of MKP-3 in the mechanisms governing persistent mechanical hypersensitivity in the peripheral tissue, as a more clinically relevant, accessible, and feasible treatment site.

This study confirms that lack of MKP-3 does not alter nociceptive behaviors in mice in response to mechanical stimulation under normal conditions. Moreover, the absence of MKP-3 does not influence the extent of acute mechanical allodynia (postoperative day 1). Nonetheless, lack of MKP-3 influences the transition from acute to chronic postoperative hypersensitivity. These data indicate that the function of MKP-3 in the peripheral injured tissue is minimal in nociceptive basal tone and the early stages of postoperative hypersensitivity. However, under normal conditions, peripheral MKP-3 seems to play a key role in the resolution phase of postsurgical mechanical allodynia by dephosphorylating p-ERK. This is supported by the temporal pattern of expression of peripheral MKP-3 and p-ERK following paw incision in WT animals. For example, expression levels of paw p-ERK decline as postoperative allodynia resolves in WT mice (postoperative days 7-12). This temporal pattern correlates well with the expression of MKP-3 in the injured tissue in these mice, which increases on postoperative days 5-7 and returns to baseline on day 12. This assumption is confirmed by the fact that the lack of MKP-3 (KO mice) results in a persistent p-ERK expression in the injured tissue. These findings show that MKP-3 controls p-ERK dephosphorylation in the incision area and 
thus promotes the resolution of postoperative hypersensitivity in acute postoperative pain.

The current study also uncovers a differential substrate preference of MKP-3 between the periphery and the spinal cord in mice. Even though MKP-3 can modulate different MAPKs, MKP-3 preferentially dephosphorylates p-ERK in most systems. ${ }^{22,23}$ It has been previously demonstrated that MKP-3 in the spinal cord modulates both p-ERK and p-p38 to a similar extent, ${ }^{7}$ and furthermore that MKP-3 preferentially dephosphorylates m-JNK (another major MAPK) in the rat brain-derived astrocytes. ${ }^{24}$ Together, these data strongly suggest that the substrate preference of MKP-3 is determined by the relevance of a given MAPK in a given tissue. This is consistent, for example, with the prominent role of $\mathrm{p}$-JNK in astrocytic function. ${ }^{35,36}$ Herein, this study observed that the temporal pattern of expression of peripheral p-p38 following paw incision was not altered in MKP-3 KO mice when compared to WT mice. In other words, p-p38 is dephosphorylated at the time of resolution of postoperative allodynia even in the absence of MKP-3. Therefore, the current results demonstrate that MKP-3 preserves its selectivity for $\mathrm{p}$-ERK at the periphery, since the lack of MKP-3 (KO mice) resulted in persistent expression of p-ERK that paralleled the persistent mechanical hypersensitivity in these mice. This study confirms the functional association between the lack of MKP-3, persistent p-ERK expression in the peripheral injured tissue, and the maintenance of postoperative mechanical hypersensitivity using an MEK inhibitor. Accordingly, phosphorylation of ERK in the peripheral tissues during inflammation contributes to the pain-related behaviors in several rodent models. ${ }^{37-39}$ Since the findings indicate that persistent $\mathrm{p}$-ERK expression on postoperative day 12 is not associated with cell infiltration (resolved at this time point), these data suggest that this MAPK phosphorylation takes place in non-immune cells, namely nerve fibers or skin cells. It has been demonstrated that an elevation of p-ERK expression in epidermal and dermal peripheral nociceptors (C-fibers) is responsible for capsaicin-induced thermal and mechanical hypersensitivity. ${ }^{40,41}$ An increase in p-ERK-1/2 and p-p38 expression as well as a functional association between the two have been observed in keratinocytes of psoriatic lesions in humans $\mathrm{s}^{42-44}$ and in mouse keratinocytes under pruritogenic conditions. ${ }^{45}$ Furthermore, p-ERK expression is enhanced in human keratinocytes in in vitro models of acute and chronic wounds. ${ }^{46,47}$ The phosphorylation of ERK can also be induced by pro-algesic factors such as epidermal growth factor. ${ }^{47}$ Since ERK phosphorylation induces the production of pro-algesic factors by non-neuronal cells that sensitize peripheral nociceptors, which in turn are characterized by neuronal ERK phosphorylation, ${ }^{6}$ it is likely that the results are explained by a pronociceptive feedback loop of skin cell and peripheral nociceptor ERK phosphorylation.

The findings of this study indicate that, although some aspects of the neuroinflammatory process might resolve in a timely fashion, following a peripheral insult, they might not be indicative of a complete resolution of the peripheral mechanisms underlying the transition from acute to chronic pain. It is plausible that the magnitude of the early cellular infiltration contributes to the persistent elevation of p-ERK in the periphery (nociceptors and/or skin cells) and subsequently to the transition from acute to chronic pain in MKP-3 KO mice. Immunohistochemistry staining to determine the specific repertoire of immune cells might better elucidate the role of immune cell infiltration in the resolution of postoperative pain. Whether the MAPK/MKP signaling pathway pattern could be used as a biomarker to identify potential patients at risk of developing chronic postoperative pain, or those already suffering from it, remains to be studied. It is certainly a testable hypothesis (minimal required invasiveness for assessment) that deserves further study. These studies in human tissue would move forward the field to set a rationale for potential studies with already available p-ERK inhibitors (some already evaluated in clinical trials for osteoarthritis or cancer). ${ }^{48,49}$

This study also suggests that p-ERK, even though it promotes a cellular pro-inflammatory phenotype, does not seem to play a role in cellular infiltration. On the other hand, p-p38 seems to be functionally associated with the initiation and resolution of cell infiltration, because of the similar pattern of appearance of these phenomena in both WT and MKP-3 KO groups. These hypotheses are in line with previous observations, in which the intraarticular inhibition of p-p38 or p-JNK, but not p-ERK, reduced cellular infiltration into the synovium, in a carrageenan-induced arthritis rat model. ${ }^{50}$ Therefore, this study does not rule out the potential role of peripheral p-p38 in the transition from acute to chronic pain. Similarly, the role of MKP-1 (which preferentially dephosphorylates p-p38) in the peripheral mechanisms of chronic pain cannot be ruled out. Elevated MKP-1 expression in MKP-3 KO mice on postoperative day 1 could suggest a compensatory mechanism triggered by surgical incision and the initial events of postoperative hypersensitivity. The high levels of p-p38 expression in the presence of elevated 
MKP-1 expression in the early stages of acute postoperative pain in MKP-3 KO animals suggest that other factors, besides MKP-1, might be involved in p38 regulation. Based on the observation that p-p38 dephosphorylation associates with the resolution of inflammation, it is speculated that inflammatory mediators could be involved in p38 modulation.

The role of MAPKs, such as p-ERK, in chronic pain models is well recognized. ${ }^{6,51}$ Activation of MAPKs is required for effective production of pro-algesic factors that sensitize peripheral nociceptors and subsequently perpetuate MAPK phosphorylation, leading to central sensitization and establishment of chronic pain. ${ }^{27}$ The induction of an effective MKP activity could break this cycle and interrupt the molecular signals driving the transition to chronic pain. It has been demonstrated that novel approaches could be utilized to develop gene therapies, for example, induction of MKP expression to modulate cell phenotype and treat chronic pain. ${ }^{27}$ The induction of MKPs can also be achieved by pharmacological approaches. For example, glucocorticoids $^{52}$ or cannabinoids ${ }^{26}$ produce anti-inflammatory effects by inducing MKP-1 and/or MKP-3. Curiously, the use of glucocorticoids in surgical patients seems to reduce the use of analgesics in postoperative pain. ${ }^{53,54}$

Although this study focused on peripheral MKP-3 and MAPK molecular changes in the context of postoperative pain resolution, it recognizes that multiple factors, such as genetics, ${ }^{55,56}$ preoperative pain and physiological states, ${ }^{57}$ psychological vulnerability, ${ }^{58}$ and surgical techniques, ${ }^{59}$ contribute to post-surgical pain and its persistence. It warrants further investigation on the potential association of these factors with the abovementioned pathways.

\section{Conclusion}

This study revealed that MKP-3/p-ERK-1/2 signaling in the periphery greatly influences the transition from acute to chronic postoperative pain. The current data further support the notion that biochemical and regulatory properties of MKP-3 render it a promising target for preventing and treating chronic postoperative pain. Moreover, studies by the authors uncover the possibility of intervening at the site of surgery, which provides a more feasible therapeutic site than the central nervous system. ${ }^{60}$

\section{Acknowledgments}

This study was supported by the Rita Allen Foundation \& American Pain Society 2011 Pain Grant (EAR-S), Hitchcock Foundation Award 2011-2012 (EAR-S), and James O
Freedman Undergraduate Presidential Scholars Program (BSL). The authors thank Ms Beth Wilkerson, Presbyterian College School of Pharmacy, for edits and revision.

\section{Disclosure}

The authors report no conflicts of interest in this work.

\section{References}

1. Macrae WA. Chronic post-surgical pain: 10 years on. Br J Anaesth. 2008;101(1):77-86.

2. Wu CL, Raja SN. Treatment of acute postoperative pain. Lancet. 25 2011;377(9784):2215-2225.

3. Alkaitis MS, Solorzano C, Landry RP, Piomelli D, DeLeo JA, RomeroSandoval EA. Evidence for a role of endocannabinoids, astrocytes and p38 phosphorylation in the resolution of postoperative pain. PLoS One. 2010;5(5):e10891.

4. Wen YR, Suter MR, Ji RR, et al. Activation of p38 mitogen-activated protein kinase in spinal microglia contributes to incision-induced mechanical allodynia. Anesthesiology. 2009;110(1):155-165.

5. Tong SE, Daniels SE, Black P, Chang S, Protter A, Desjardins PJ. Novel p38alpha mitogen-activated protein kinase inhibitor shows analgesic efficacy in acute postsurgical dental pain. J Clin Pharmacol. 2012; 52(5):717-728.

6. Ji RR, Gereau RWt, Malcangio M, Strichartz GR. MAP kinase and pain. Brain Res Rev. 2009;60(1):135-148.

7. Saha M, Skopelja S, Martinez E, Alvarez DL, Liponis BS, RomeroSandoval EA. Spinal mitogen-activated protein kinase phosphatase-3 (MKP-3) is necessary for the normal resolution of mechanical allodynia in a mouse model of acute postoperative pain. J Neurosci. 2013; 33(43): 17182-17187.

8. Huang L, Gao YJ, Wang J, Strichartz G. Shifts in cell-type expression accompany a diminishing role of spinal p38-mapkinase activation over time during prolonged postoperative pain. Anesthesiology. 2011; 115(6):1281-1290.

9. Ostenfeld T, Krishen A, Lai RY, et al. Analgesic efficacy and safety of the novel p38 MAP kinase inhibitor, losmapimod, in patients with neuropathic pain following peripheral nerve injury: a double-blind, placebo-controlled study. Eur J Pain. 2013;17(6):844-857.

10. Ostenfeld T, Krishen A, Lai RY, et al. A randomized, placebo-controlled trial of the analgesic efficacy and safety of the p38 MAP kinase inhibitor, losmapimod, in patients with neuropathic pain from lumbosacral radiculopathy. Clin J Pain. 2015;31(4):283-293.

11. Lin X, Wang M, Zhang J, Xu R. p38 MAPK: a potential target of chronic pain. Curr Med Chem. 2014;21(38):4405-4418.

12. Spofford CM, Brennan TJ. Gene expression in skin, muscle, and dorsal root ganglion after plantar incision in the rat. Anesthesiology. 2012;117(1):161-172.

13. Wu C, Erickson MA, Xu J, Wild KD, Brennan TJ. Expression profile of nerve growth factor after muscle incision in the rat. Anesthesiology. 2009;110(1):140-149.

14. Kim TJ, Freml L, Park SS, Brennan TJ. Lactate concentrations in incisions indicate ischemic-like conditions may contribute to postoperative pain. J Pain. 2007;8(1):59-66.

15. Kroin JS, Buvanendran A, Watts DE, Saha C, Tuman KJ. Upregulation of cerebrospinal fluid and peripheral prostaglandin $\mathrm{E} 2$ in a rat postoperative pain model. Anesth Analg. 2006;103(2):334-343, table of contents.

16. Jang JH, Clark JD, Li X, Yorek MS, Usachev YM, Brennan TJ. Nociceptive sensitization by complement $\mathrm{C} 5 \mathrm{a}$ and $\mathrm{C} 3 \mathrm{a}$ in mouse. Pain. 2010;148(2):343-352.

17. Ferreira J, Triches KM, Medeiros R, Calixto JB. Mechanisms involved in the nociception produced by peripheral protein kinase $\mathrm{c}$ activation in mice. Pain. 2005;117(1-2):171-181. 
18. Holzheimer RG, Steinmetz W. Local and systemic concentrations of pro- and anti-inflammatory cytokines in human wounds. Eur J Med Res. 2000;5(8):347-355

19. De Jongh RF, Vissers KC, Meert TF, Booij LH, De Deyne CS, Heylen RJ. The role of interleukin-6 in nociception and pain. Anesth Analg 2003;96(4):1096-1103, table of contents.

20. Melemedjian OK, Asiedu MN, Tillu DV, et al. IL-6- and NGF-induced rapid control of protein synthesis and nociceptive plasticity via convergent signaling to the eIF4F complex. J Neurosci. 2010;30(45): 15113-15123.

21. Tillu DV, Melemedjian OK, Asiedu MN, et al. Resveratrol engages AMPK to attenuate ERK and mTOR signaling in sensory neurons and inhibits incision-induced acute and chronic pain. Mol Pain. 2012;8:5.

22. Kim Y, Rice AE, Denu JM. Intramolecular dephosphorylation of ERK by MKP3. Biochemistry. 2003;42(51):15197-15207.

23. Owens DM, Keyse SM. Differential regulation of MAP kinase signalling by dual-specificity protein phosphatases. Oncogene. 2007; 26(22):3203-3213

24. Ndong C, Landry RP, Saha M, Romero-Sandoval EA. Mitogenactivated protein kinase (MAPK) phosphatase-3 (MKP-3) displays a p-JNK-MAPK substrate preference in astrocytes in vitro. Neurosci Lett. 2014;575:13-18.

25. Romero-Sandoval EA, Horvath R, Landry RP, DeLeo JA. Cannabinoid receptor type 2 activation induces a microglial anti-inflammatory phenotype and reduces migration via MKP induction and ERK dephosphorylation. Mol Pain. 2009;5:25.

26. Landry RP, Martinez E, DeLeo JA, Romero-Sandoval EA. Spinal cannabinoid receptor type 2 agonist reduces mechanical allodynia and induces mitogen-activated protein kinase phosphatases in a rat model of neuropathic pain. J Pain. 2012;13(9):836-848.

27. Ndong C, Landry RP, DeLeo JA, Romero-Sandoval EA. Mitogen activated protein kinase phosphatase-1 prevents the development of tactile sensitivity in a rodent model of neuropathic pain. Mol Pain. 2012; $8: 34$

28. Wang X, Meng X, Kuhlman JR, et al. Knockout of Mkp-1 enhances the host inflammatory responses to gram-positive bacteria. J Immunol. 2007;178(8):5312-5320.

29. Salojin K, Oravecz T. Regulation of innate immunity by MAPK dualspecificity phosphatases: knockout models reveal new tricks of old genes. J Leukoc Biol. 2007;81(4):860-869.

30. Maillet M, Purcell NH, Sargent MA, York AJ, Bueno OF, Molkentin JD. DUSP6 (MKP3) null mice show enhanced ERK1/2 phosphorylation at baseline and increased myocyte proliferation in the heart affecting disease susceptibility. J Biol Chem. 2008;283(45):31246-31255.

31. Reddyjarugu B, Pavek T, Southard T, Barry J, Singh B. Analgesic efficacy of firocoxib, a selective inhibitor of cyclooxygenase 2, in a mouse model of incisional pain. J Am Assoc Lab Animal Sci. 2015; 54(4):405-410.

32. Pogatzki EM, Raja SN. A mouse model of incisional pain. Anesthesiology. 2003;99(4):1023-1027.

33. Chaplan SR, Bach FW, Pogrel JW, Chung JM, Yaksh TL. Quantitative assessment of tactile allodynia in the rat paw. $J$ Neurosci Methods. 1994;53(1):55-63.

34. Karim F, Wang CC, Gereau RWt. Metabotropic glutamate receptor subtypes 1 and 5 are activators of extracellular signal-regulated kinase signaling required for inflammatory pain in mice. J Neurosci. 2001;21(11):3771-3779.

35. Gao YJ, Zhang L, Samad OA, et al. JNK-induced MCP-1 production in spinal cord astrocytes contributes to central sensitization and neuropathic pain. J Neurosci. 2009;29(13):4096-4108.

36. Zhuang ZY, Wen YR, Zhang DR, et al. A peptide c-Jun N-terminal kinase (JNK) inhibitor blocks mechanical allodynia after spinal nerve ligation: respective roles of $\mathrm{JNK}$ activation in primary sensory neurons and spinal astrocytes for neuropathic pain development and maintenance. J Neurosci. 2006;26(13):3551-3560.
37. Hao J, Liu MG, Yu YQ, et al. Roles of peripheral mitogen-activated protein kinases in melittin-induced nociception and hyperalgesia. Neuroscience. 2008;152(4):1067-1075.

38. Mao Q, Ruan J, Cai X, et al. Antinociceptive effects of analgesicantitumor peptide (AGAP), a neurotoxin from the scorpion Buthus martensii Karsch, on formalin-induced inflammatory pain through a mitogen-activated protein kinases-dependent mechanism in mice. PLoS One. 2013;8(11):e78239.

39. Kassuya CA, Ferreira J, Claudino RF, Calixto JB. Intraplantar PGE2 causes nociceptive behaviour and mechanical allodynia: the role of prostanoid $\mathrm{E}$ receptors and protein kinases. Br J Pharmacol. 2007;150(6):727-737.

40. DaiY, Iwata K, Fukuoka T, et al. Phosphorylation of extracellular signal-regulated kinase in primary afferent neurons by noxious stimuli and its involvement in peripheral sensitization. J Neurosci. 2002;22(17):7737-7745.

41. Zhuang ZY, Xu H, Clapham DE, Ji RR. Phosphatidylinositol 3-kinase activates ERK in primary sensory neurons and mediates inflammatory heat hyperalgesia through TRPV1 sensitization. J Neurosci. 2004;24(38):8300-8309.

42. Johansen C, Kragballe K, Westergaard M, Henningsen J, Kristiansen K, Iversen L. The mitogen-activated protein kinases p38 and ERK1/2 are increased in lesional psoriatic skin. Br J Dermatol. 2005;152(1): 37-42.

43. Yu XJ, Li CY, Dai HY, et al. Expression and localization of the activated mitogen-activated protein kinase in lesional psoriatic skin. Exp Mol Pathol. 2007;83(3):413-418.

44. Soegaard-Madsen L, Johansen C, Iversen L, Kragballe K. Adalimumab therapy rapidly inhibits $\mathrm{p} 38$ mitogen-activated protein kinase activity in lesional psoriatic skin preceding clinical improvement. Br J Dermatol. 2010;162(6):1216-1223.

45. Chen Y, Fang Q, Wang Z, et al. Transient receptor potential vanilloid 4 ion channel functions as a pruriceptor in epidermal keratinocytes to evoke histaminergic itch. J Biol Chem. 2016;291(19):10252-10262.

46. Alcaraz A, Mrowiec A, Insausti CL, et al. Amniotic membrane modifies the genetic program induced by TGFss, stimulating keratinocyte proliferation and migration in chronic wounds. PLoS One. 2015; 10(8):e0135324.

47. Gandham VD, Maddala RL, Rao V, et al. Effects of Y27632 on keratinocyte procurement and wound healing. Clin Exp Dermatol. 2013; 38(7):782-786.

48. Loeser RF, Erickson EA, Long DL. Mitogen-activated protein kinases as therapeutic targets in osteoarthritis. Curr Opin Rheumatol. 2008;20(5):581-586.

49. LoRusso PM, Krishnamurthi SS, Rinehart JJ, et al. Phase I pharmacokinetic and pharmacodynamic study of the oral MAPK/ERK kinase inhibitor PD-0325901 in patients with advanced cancers. Clin Cancer Res. 2010;16(6):1924-1937.

50. Han H, Lee KS, Rong W, Zhang G. Different roles of peripheral mitogen-activated protein kinases in carrageenan-induced arthritic pain and arthritis in rats. Anesth Analg. 2012;115(5):1221-1227.

51. Ji RR, Xu ZZ, Gao YJ. Emerging targets in neuroinflammation-driven chronic pain. Nat Rev Drug Discov. 2014;13(7):533-548.

52. EngelbrechtY, de Wet H, Horsch K, Langeveldt CR, Hough FS, Hulley PA. Glucocorticoids induce rapid up-regulation of mitogen-activated protein kinase phosphatase-1 and dephosphorylation of extracellular signal-regulated kinase and impair proliferation in human and mouse osteoblast cell lines. Endocrinology. 2003;144(2):412-422.

53. Waldron NH, Jones CA, Gan TJ, Allen TK, Habib AS. Impact of perioperative dexamethasone on postoperative analgesia and side-effects: systematic review and meta-analysis. Br J Anaesth. 2013;110(2):191-200.

54. Shaikh S VH, Yadav N, Jauhari M, Bullangowda J. Applications of steroid in clinical practice: a review. SRN Anesthesiol. 2012;(2012):11.

55. Devor M, Raber P. Heritability of symptoms in an experimental model of neuropathic pain. Pain. 1990;42(1):51-67.

56. Mogil JS. The genetic mediation of individual differences in sensitivity to pain and its inhibition. Proc Natl Acad Sci USA. 1999;96(14):7744-7751. 
57. Sommer M, de Rijke JM, van Kleef M, et al. Predictors of acute postoperative pain after elective surgery. Clin J Pain. 2010;26(2): 87-94.

58. Peters ML, Sommer M, de Rijke JM, et al. Somatic and psychologic predictors of long-term unfavorable outcome after surgical intervention. Ann Surg. 2007;245(3):487-494.
59. Kehlet H, Jensen TS, Woolf CJ. Persistent postsurgical pain: risk factors and prevention. Lancet. 2006;367(9522):1618-1625.

60. Klongnoi B, Kaewpradub P, Boonsiriseth K, Wongsirichat N. Effect of single dose preoperative intramuscular dexamethasone injection on lower impacted third molar surgery. Int J Oral Maxillofac Surg. 2012;41(3):376-379.

\section{Publish your work in this journal}

The Journal of Pain Research is an international, peer reviewed, open access, online journal that welcomes laboratory and clinical findings in the fields of pain research and the prevention and management of pain. Original research, reviews, symposium reports, hypothesis formation and commentaries are all considered for publication.
Dovepress

The manuscript management system is completely online and includes a very quick and fair peer-review system, which is all easy to use. Visit http://www.dovepress.com/testimonials.php to read real quotes from published authors. 